\title{
COMPARACIÓN ENTRE EL MODELO PROBIT Y EL MODELO DE HILL PARA DETERMINAR LA EFICACIA DE IVERMECTINA SOBRE LARVAS DE Rhipicephalus (Boophilus) microplus (ACARI, IXODIDA: IXODIDAE) MEDIANTE LA TÉCNICA DE INMERSIÓN.
}

\author{
Torrents $\mathrm{J}^{1}$, Formentini $\mathrm{E}^{2}$, Nava $\mathrm{S}^{3}$
}

\author{
${ }^{1}$ Cátedra de Zoología, FCV-UNL \\ ${ }^{2}$ Laboratorio de Farmacología y Toxicología, FCV-UNL \\ ${ }^{3}$ Instituto Nacional de Tecnología Agropecuaria (INTA EEA Rafaela)
}

* Correspondencia: Torrents J, E-mail: torrentsjorgelina@gmail.com

COMPARISON BETWEEN THE PROBIT MODEL AND THE HILL MODEL TO DETERMINE THE EFFICACY OF IVERMECTIN ON Rhipicephalus (Boophilus) microplus (ACARI, IXODIDA: IXODIDAE) WITH LARVAL IMMERSION TECHNIQUE

\section{SUMMARY}

The aim of this study was to propose an alternative statistic treatment for the results obtained by testing the in vitro efficacy of ivermectin (IVM) against $R$. microplus using the larval immersion test in order to detect the emergence of resistant strains. The statistical analysis of the results obtained by this technique is standardized by the FAO who proposes the use of the Probit model. However there are alternative mathematical models that allow a more precise analysis of experimental data generating more information as nonlinear logistic equation, also called the Hill model. The results of the IVM efficacy against a sensible strain of $R$. microplus were analysed whit both model. A comparison between the results obtained with PROBIT and Hill model showed that the last one was able to make a more precise fit of the experimental data, generating more information about small IVM concentrations. The C50 (13.58 ppm) obtained with Probit model result smaller than the obtained with the Hill model (14.24 ppm), however the C90, C99 and the discriminant dose (19.31 ppm, 25.74 ppm, 51.48 ppm) obtained with de Hill model were bigger than the obtained with the Probit model (17.50 ppm, 21.98 ppm, $43.96 \mathrm{ppm})$.

Palabras clave: Rhipicephalus microplus, test de inmersión de larvas, análisis estadistico.

Keywords: Rhipicephalus microplus, larval immersion test, statistical analysis.

Rhipicephalus (Boophilus) microplus vulgarmente conocida como garrapata común del bovino, es el ectoparásito hematófago de mayor importancia a nivel mundial para la producción pecuaria bovina. En Argentina este ectoparásito se encuentra distribuido en la zona norte al paralelo 30 -31으, S, produciendo grandes pérdidas directas e indirectas en la producción, además de ser vector exclusivo de Babesia bigemina y $B$. bovis.
El método de control más utilizado en la Argentina es el empleo de garrapaticidas químicos, cuyo uso indiscriminado ha derivado en la aparición de resistencia a las drogas arsenicales, organofosforados, piretroides, formamidinas y fenilpirazoles. También es posible que la resistencia ya se encuentre establecida para drogas como ivermectina (IVM), fluazurón y fipronil (Nava et al., 2018). El diagnostico de resistencia in vitro es una 
herramienta útil para conocer el estado actual de sensibilidad a los principios activos empleados para su control, además de permitir elaborar estrategias de tratamiento en función de los principios químicos que aún se encuentran activos en cada establecimiento. Las técnicas in vitro para determinar la sensibilidad y la resistencia de este ectoparásito a los principios activos en uso se hallan estandarizadas por la FAO (FAO, 2004), proponiendo el empleo del modelo PROBIT para el ajuste de los datos de eficacia en función de concentraciones crecientes de los principios activos en estudio y el posterior cálculo de las concentraciones efectivas al 50\% (C50), 90\% (C90) y 99\% (C99). El modelo PROBIT se basa en transformar los datos experimentales (log concentraciones y \% eficacia expresados como unidades PROBIT) de manera que los mismos puedan ser analizados con una función lineal de primer grado o ecuación de la recta. Actualmente se dispone de softwares capaces de analizar los datos directamente sin linearización usando modelos no lineares como el modelo sigmoideo de respuesta máxima o de HILL (Goutelle et al., 2008). En este ensayo se estudió la actividad de IVM sobre una cepa sensible de R. microplus (0.17) identificada como $S$ mediante la técnica de inmersión de larvas (TLI), utilizando un estándar de IVM de pureza conocida (Sigma-Adrich) en un intervalo de concentraciones comprendido entre 0 y $50 \mathrm{ppm}$. El recuento de larvas vivas y muertas se realizó a las $24 \mathrm{~h}$ y los resultados de la curva concentración (ppm)-eficacia (\% muertas) fueron ajustados con el modelo PROBIT (LeOra Software, 2003) y con el modelo sigmoideo de respuesta máxima o de HILL mediante regresión no lineal ponderada de mínimos cuadrados utilizando el software ADAPT II (BMRS, University of Southern California, USA. El modelo de HILL es descripto como: $\mathrm{E}=\mathrm{EO}+[(\mathrm{Emax}$ $. \mathrm{CN}) /(\mathrm{C} 5 \mathrm{ON}+\mathrm{CN})]$, donde $\mathrm{E}$ es el efecto expresado en \%, EO es el efecto basal, Emax es el efecto máximo, C es la concentración, C50 es la concentración que logra el 50\% de Emax y $\mathrm{N}$ es el coeficiente de sigmoidicidad o de HILL (Goutelle et al., 2008). La discriminación del ajuste obtenido con los dos modelos se realizó por: (i) inspección visual de los datos observados y estimados por los mismos, (ii) inspección visual de la dispersión al azar de las residuales alrededor de los valores estimados y (iii) evaluación de los valores de la sumatoria del cuadrado de las residuales (SSR). Los valores de concentración efectiva al 50\% (C50), al 90\% (C90) y al 99\% (C99) se estimaron con ambos modelos. La dosis discriminante (DD) de IVM para cada modelo se estimó como C99 × 2. El ajuste de los datos experimentales $(\%$ eficacia) en función de las concentraciones de IVM (ppm) sobre larvas de la cepa $S(0.17)$ de $R$. microplus y las gráficas de la dispersión de las residuales versus el eje de las abscisas obtenidas con el modelo PROBIT y con el modelo de HILL se presentan en la figura 1.

Los parámetros de ajuste estimados con el modelo sigmoideo de respuesta máxima o de HILL se presentan en la tabla 1.
Los valores de SSR, las C50, C90 y C99 (ppm) de IVM estimadas a partir de los resultados obtenidos con el modelo PROBIT y el modelo de HILL y la DD estimada a partir de los resultados obtenidos con ambos modelos se presentan en la tabla 2.

Los resultados obtenidos en este estudio mostraron que el análisis de los datos experimentales con el modelo de HILL proporcionó información acerca de una actividad basal (EO) observada a bajas concentraciones de IVM (12,83\%), fenómeno que no pudo ser discriminado por el modelo PROBIT. Por otra parte, el valor de $N>1(9,81)$, indica que IVM presenta sobre las larvas de R. microplus una actividad de tipo todo o nada. El valor adimensional de $\mathrm{N}$ es más fácil de interpretar que la pendiente generada por el modelo PROBIT, y podría ser utilizado para discriminar cepas de larvas con distinta sensibilidad a la respuesta de las concentraciones de IVM. Respecto de la bondad de ajuste obtenida con los modelos PROBIT y de HILL podemos concluir que para este último se obtuvo un mejor ajuste (Figura $1 \mathrm{~A}$ y C), una dispersión de residuales más cercana al valor estimado (Figura $1 \mathrm{~B}$ y D) y menores valores de SSR (Tabla 2). Estos resultados mostraron que el modelo de HILL permitiría estimar valores de C50, C90 y C99 más robustos y precisos que los que se obtendrían con el modelo PROBIT, permitiendo estimar una DD más precisa. Un mayor número de aislamientos tratados, así como la incorporación de nuevas concentraciones en el protocolo de TLI permitirán obtener nuevas perspectivas sobre la interpretación de los resultados in vitro, pudiendo tener alternativas con mejor ajuste. El conocimiento de la acción de esta droga a campo nos permitirá determinar cuáles son los modelos que se ajustan de mejor forma al procesamiento de los datos obtenidos en laboratorio.
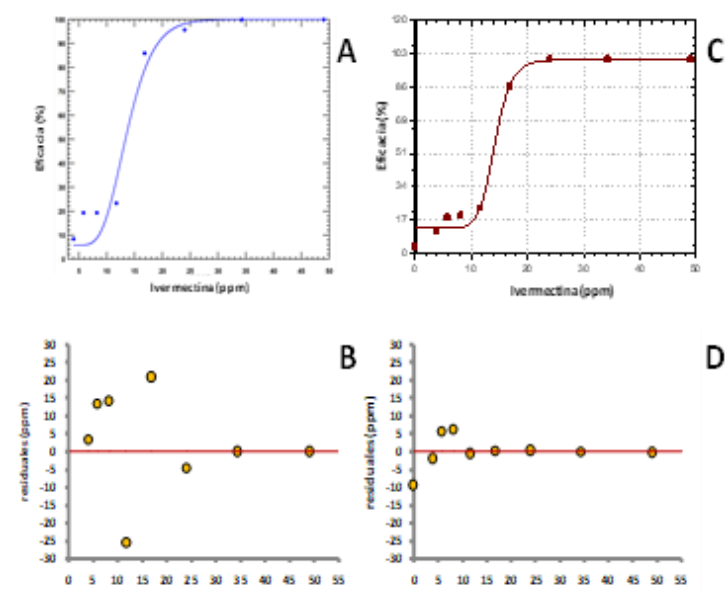

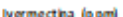

vermectra (pomi)

Figura 1. Ajuste de los datos experimentales (\% eficacia) en función de las concentraciones de IVM (ppm) sobre larvas de la cepa S (0.17) de $R$. microplus con el modelo PROBIT (A y B) y con el modelo sigmoideo de respuesta máxima o de HILL ( $C$ y D). A y $C$ corresponden al ajuste de los datos de eficacia con el modelo de PROBIT y de HILL respectivamente y $B$ y $D$ corresponden $y$ 
la dispersión de las residuales obtenidos con el modelo PROBIT y de HILL respectivamente.

Tabla 1. Parámetros estimados a partir del ajuste de los datos de eficacia y concentración de IVM con el modelo de HILL.

\begin{tabular}{lc}
\hline Parámetros & Valores \\
\hline $\mathrm{E}_{0}(\%)$ & 12,83 \\
$\mathrm{E}_{\max }$ est (\%) & 87,38 \\
$\mathrm{E}_{\max }$ real (\%) & 100,21 \\
$\mathrm{CE}_{\text {so }}$ (ppm) & 14,24 \\
$\mathrm{~N}$ & 9,81 \\
\hline
\end{tabular}

Tabla2: Sumatoria de las residuales al cuadrado (SSR), concentraciones de IVM con el $50 \%, 90 \%$ y $99 \%$ de eficacia y las dosis discriminantes (DD) estimadas con el modelo PROBIT y el modelo de HILL.

\begin{tabular}{lcc}
\hline Parámetros & Probit & Modelo de Hill \\
\hline SSR & 1503,45 & 161,63 \\
C $_{50}(\mathrm{ppm})$ & 13,58 & 14,24 \\
C $_{90}(\mathrm{ppm})$ & 19,31 & 17,50 \\
C $_{99}(\mathrm{ppm})$ & 25,74 & 21,98 \\
Dosis discriminante $(\mathrm{ppm})$ & 51,48 & 43,96 \\
\hline
\end{tabular}

\section{Bibliografía}

FAO. 2004. Guideline resistance management and integrated parasite control in ruminants. Acaricide resistance: diagnosis, management and prevention. Agr. Dept. Animal Production and Health Division. Roma, Italia. 25-77 pp.

Goutelle S, Maurin M, Rougier F, Barbaut X, Bourguignon L, Ducher M, Maire P. 2008. The Hill equation: a review of its capabilities in pharmacological modelling. Fundamental Clinical Pharmacology. 22 (6): 633-648.

Nava S, Morel N, Mangold AJ, Guglielmone AA. 2018. Un caso de resistencia de Rhipicephalus microplus (Acari: Ixodidae) al fipronil detectado en pruebas de campo en el este de Santiago del Estero. Argentina. Revista FAVE. 17: 15. 